\title{
Espaces scolaires de la rentrée
}

Architectures et cérémonies d'accueil dans trois lycées à Copenhague

School spaces at the start of the school year. Architecture and welcome ceremonies in three secondary schools in Copenhagen

Espacios escolares del inicio del año escolar. Arquitecturas y ceremonias de acogida en tres institutos de Copenhague

\section{Henrik Reeh}

\section{OpenEdition}

Journals

Édition électronique

URL : http://journals.openedition.org/ries/3611

DOI : 10.4000/ries.3611

ISSN : 2261-4265

Éditeur

Centre international d'études pédagogiques

Édition imprimée

Date de publication : 1 décembre 2013

Pagination : 65-76

ISBN : 978-2-85420-601-2

ISSN : $1254-4590$

\section{Référence électronique}

Henrik Reeh, «Espaces scolaires de la rentrée », Revue internationale d'éducation de Sèvres [En ligne], 64 | décembre 2013, mis en ligne le 01 décembre 2015, consulté le 01 mai 2019. URL : http:// journals.openedition.org/ries/3611 ; DOI : 10.4000/ries.3611 


\title{
Espaces scolaires de la rentrée
}

\section{Architectures et cérémonies d'accueil dans trois lycées à Copenhague}

\author{
Henrik Reeh
}

\section{HABITUDES, REPRÉSENTATION, RENTRÉE SCOLAIRE}

Au sein du lycée moderne, dont on se propose d'étudier trois établissements emblématiques au Danemark, les espaces scolaires se divisent en deux groupes majeurs. Il y a d'abord les espaces - les salles de classe - qui sont liés à la transmission du savoir mais aussi aux habitudes implicites de la vie pédagogique. Semblables à des chambres noires, ces espaces se trouvent hors du champ de vision d'observateurs externes; dès qu'on s'y introduit pour faire de l'observation de classe, l'ambiance en est fortement affectée. Il y a aussi des espaces de représentation - les salles des fêtes - qui servent à la mise en scène officielle du lycée devant les parents ou les élèves eux-mêmes. Dans de telles situations, tout est fait pour être vu par des yeux qui, en grande partie, sont éloignés de la vie pédagogique de tous les jours et qui n'en voient, ici, qu'un reflet idéalisé et superficiel.

Voilà comment les espaces scolaires du lycée rappellent les deux versants de la réception architecturale selon le philosophe Walter Benjamin. Dans L'euvre d'art à l'époque de sa reproductibilité technique (1936), Benjamin établit le double caractère de l'expérience architecturale de la façon suivante :

"Les édifices font l'objet d'une double réception : par l'usage et par la perception. En termes plus précis: d'une réception tactique ${ }^{1}$ et d'une réception visuelle. »(p. 108)

La duplicité relevée souligne une certaine réciprocité entre l'usage et la vision, mais elle permet aussi de comprendre comment une polarisation entre les deux registres de l'expérience spatiale peut se produire. Alors que la vision se prête à la représentation sous forme d'images, les habitudes de l'usage demeurent plutôt secrètes, difficiles à décrire et à comprendre. Ainsi, dans la double réception de l'espace notée par Benjamin, les liens entre les dimensions tactique et optique sont-ils souvent indirects et indicibles. C'est pourquoi

\footnotetext{
1. Dans les versions originales en allemand, Walter Benjamin emploie bien le terme "taktisch » (tactique), qui devient « tactile » dans la première traduction en français par Pierre Klossowski de 1936. Or « tactique » correspondrait mieux à l'usage, auquel Benjamin fait référence. Benjamin, $1989: 381,664$. Cf. Reeh, 100-102.
} 
l'unité dialectique entre l'espace visuel et les pratiques quotidiennes tend à nous échapper².

Grâce au lien substantiel entre les deux composantes de la réception architecturale, une approche concrète nous permet cependant de circonscrire des espaces scolaires qui se situent, en quelque sorte, à mi-chemin entre les extrêmes que sont les habitudes implicites, d'une part, et la représentation visuelle, de l'autre. Une situation où, justement, se mêlent l'usage et la vision, les habitudes et la représentation s'établit dans le cadre spatial où se déroule la rentrée scolaire des lycéens. Tout comme l'enseignement de tous les jours, l'accueil des élèves après les vacances d'été fait partie des traditions internes à l'établissement. Dans la mesure où l'accueil a lieu dans les espaces collectifs du lycée, il est néanmoins plus accessible, moins clos sur lui-même que ne le sont maints rituels d'apprentissage qui se cachent dans les salles de classe.

La cérémonie de la rentrée scolaire a un avantage majeur pour l'observateur externe : celui-ci peut y assister et même y photographier sans perturber le comportement des acteurs de cet événement qui, participant de la vie interne du lycée, constitue une situation où convergent l'architecture scolaire et les valeurs pédagogiques de l'établissement. Puisque les festivités de la rentrée scolaire à Copenhague ont lieu à différentes dates, l'auteur du présent article a pu assister aux séances d'accueil dans l'ensemble des trois lycées qui vont nous aider à considérer les espaces scolaires comme une coexistence de cadres physiques et de processus humains ${ }^{3}$.

Dessinés par des architectes danois de renom, les lycées sélectionnés ont été achevés à trois moments de l'histoire moderne : en 1924, en 1972 et en 2007. Bien entretenus et modernisés, ils sont toujours à la hauteur de l'agenda pédagogique qui, pourtant, a profondément changé depuis l'entre-deux-guerres, où $4 \%$ des jeunes fréquentaient le lycée, jusqu'à aujourd'hui où plus de $40 \%$ obtiennent leur baccalauréat. D’ailleurs, les trois établissements sont reconnus pour leurs réponses pédagogiques aux sérieux défis qui, au début du XXI siècle, se présentent à l'institution du lycée général. Au-delà des conditions sociogéographiques qui influent sur le recrutement des élèves, les espaces scolaires des trois cérémonies d'accueil font donc entrevoir différentes stratégies pour le lycée de l'avenir. C'est donc à l'analyse des espaces scolaires de la rentrée lycéenne - catégorie intermédiaire entre les espaces scolaires de la routine et ceux de la représentation - que l'étude suivante va se consacrer.

3. La rentrée scolaire 2013 a eu lieu le 13 août au lycée Sankt Annæ ; le 15 août au lycée Ørestad ; le 19 août au lycée Øregård. Pour respecter les différences de chronologie, de typologie et de pédagogie, la présentation ci-dessous suivra l'ordre d'achèvement des trois bâtisses scolaires : lycée Øregård (1924), lycée Sankt Annæ (1972), lycée Ørestad (2007). 


\section{AULA ET DISCOURS RECTORAL AU LYCEE ØREGÅRD}

Établissement de référence pour son architecture par Edvard Thomsen et G. B. Hagen ${ }^{4}$ mais aussi pour ses anciens élèves - dont l'actuel Prince héritier de la couronne danoise -, le lycée Øregård (Øregård [Oeregaard] Gymnasium) se situe dans le quartier aisé de Hellerup, entouré de belles villas et de verdure ${ }^{5}$. Une visite à l'établissement donne à voir un contraste entre, d'un côté, l'extérieur néoclassique et symétrique d'un bâtiment austère et, de l'autre, son intérieur clair et presque chaleureux dominé par un grand hall central au toit de verre, l'aula. Grande fierté du lycée, cette aula est plus qu'une salle des fêtes: elle constitue le centre autour duquel l'ensemble des couloirs et des salles de classe s'articule sur plusieurs niveaux. La sculpture Jason et la Toison d'or œuvre maîtresse de Bertil Thorvaldsen (1770-1844) - et une tribune portant l'inscription ARS LONGA / VITA BREVIS, située devant une plaque commémorative énumérant les personnes tombées pendant l'Occupation allemande, assurent à cet espace à échelle humaine son ambiance sobre et classique.

C'est au sein de l'aula que se rassemblent les professeurs et les élèves une fois par semaine pour le cantique du matin; de même, c'est ici qu'ont lieu les réunions et les spectacles publics qui rythment l'année scolaire. En temps

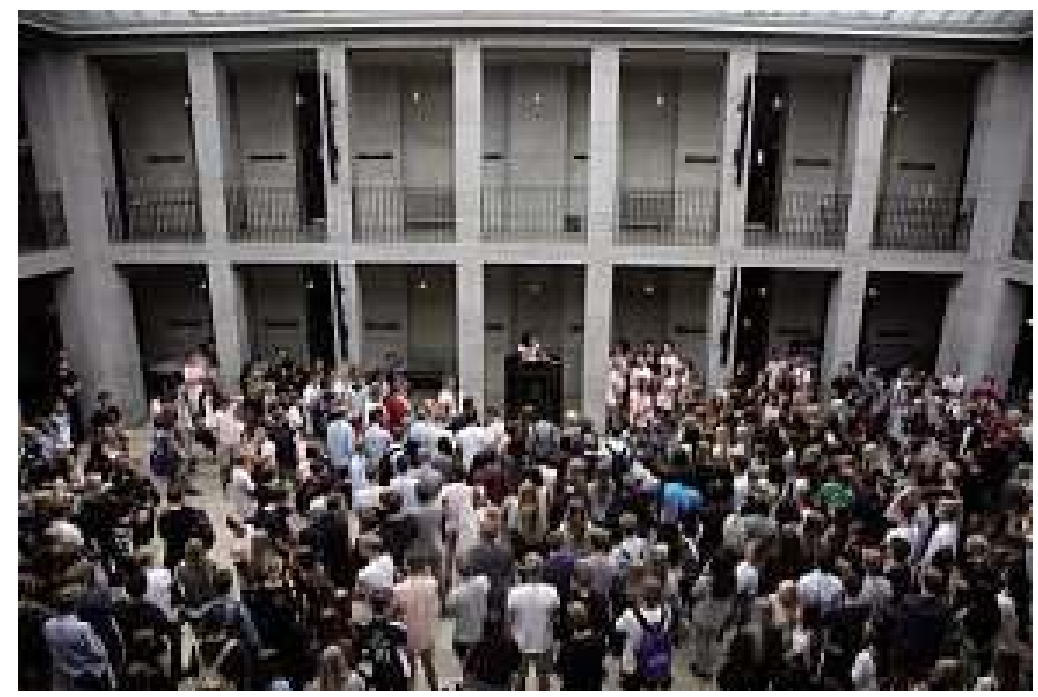

Photo 1.

Aula du lycée Øregård. Rentrée 2013 (Photo : Henrik Reeh)

4. Balslev Jørgensen, 140-141. Sur d'autres écoles à aula par l'architecte Edvard Thomsen, voir Coninck-Smith, 2011, 23, 112-115.

5. Askgaard \& Haugan, 2003. 
ordinaire, des tables et des fauteuils invitent les élèves à s'installer pour travailler. Aujourd'hui, jour de la rentrée, il n'y a ni tables ni chaises dans le hall, qui se remplit de jeunes gens aux cheveux blonds et habillés de vêtements de marque. Ce n'est pas pour rien que les élèves eux-mêmes parlent du trottoir allant du lycée à la gare en termes de catwalk: le podium d'un défilé de mode. Les mentors des nouveaux élèves sont facilement reconnaissables par leur T-shirt rose, qui résume l'optimisme social de cette jeunesse qui, en optant pour une scolarité à Øregård, se retrouve dans un lycée bien ancré dans le monde des affaires.

La proviseure, bronzée et dynamique, avait prévenu que son allocution serait brève : un simple mot de bienvenue. Il s'agit tout de même d'un véritable discours, cohérent et mobilisateur, qui encourage les élèves à s'engager dans la vie commune du lycée. À cet effet, la proviseure attire l'attention sur une bannière colorée, suspendue entre deux colonnes de la galerie supérieure: «Engage-toi dans [la vie d'] Øregård ». Ainsi invite-t-elle les jeunes à s'approprier leur lycée : «Bienvenue à votre Øregård » et "Sans toi, aucun Øregård », annonçaient déjà des bannières à l'entrée de l'établissement.

Si la proviseure d'Øregård incite bien les jeunes à s'engager dans la vie communautaire, la cérémonie d'accueil dans l'aula n'invite pas pour autant les élèves à sortir d'une position d'écoute et de consentement. Les jeunes sont debout dans le hall pendant la dizaine de minutes que dure cette assemblée.

Le programme de la rentrée continue ailleurs; bientôt l'aula sera vide et silencieuse, prête à être admirée pour ses qualités plastiques et lumineuses. Ceci est bien une œuvre d'architecture qui n'a guère perdu de sa puissance après un siècle. Cependant, ce hall commun qui se laisse aujourd'hui traverser dans tous les sens, n'a pas toujours été d'accès libre. Il a fallu attendre 1968 pour que les élèves aient le droit d'y passer sans autorisation ${ }^{6}$.

Malgré sa quasi-absence d'ornements classiques, le bâtiment du lycée Øregård figure en bonne position dans la lignée néoclassique qui parcourt l'architecture danoise - il y est cité aux côtés de chefs-d'œuvre comme l'église royale Vor Frue Kirke (l'église Notre Dame, 1829) ou la préfecture de police de Copenhague (1924). Or le bâtiment d'Øregård est aussi un monument fort dans l'histoire de l'architecture scolaire, annonçant une modernité démocratique dans laquelle les espaces collectifs visent à promouvoir la communauté des élèves et des professeurs.

\section{Salle de concert et voix DU COLLECTIF AU LYCÉE SAINTE-Anne}

Sans être un établissement inconnu, le lycée Sainte Anne (Sankt Annoe Gymnasium) occupe une position vague sur la carte urbaine et architecturale de

6. Askgaard \& Haugan, 2011, 71-73. 
la capitale danoise. Construit au début des années 1970, à la fin des Trente Glorieuses, cet établissement est l'œuvre de Preben Hansen, l'architecte de l'Ambassade de Danemark à Paris. Dominé par des immeubles HLM et un grand cimetière, le quartier fait penser à la banlieue plutôt qu'à la ville. Néanmoins, ce lycée n'est qu'à trois stations de la gare centrale et il s'inscrit dans une infrastructure de transports en commun qui permet aux élèves de venir de toute la région métropolitaine pour y suivre un cursus imprégné de musique.

L'architecture fonctionnaliste de cet établissement ne suscite plus l'enthousiasme des élèves et des professeurs. Or il n'échappe à personne qu'en plus de sa propre piscine, ce lycée étendu et un peu anonyme possède une salle de concert de plus de mille places assises. Avec une hauteur de dix à douze mètres sous plafond, le parterre couvre plus de 600 mètres carrés, auxquels s'ajoutent un grand balcon avec ses dix rangées de places supplémentaires. Équipée d'une scène surélevée et d'un orgue à 1124 tuyaux, cette salle aux murs en brique jaune et aux reliefs sculpturaux, couverts de feuilles d'or exhibe une monumentalité que l'on peine à associer à un établissement scolaire financé par une municipalité peu fortunée. Or le lycée Sainte-Anne, qui comporte aussi "L'école de chant " (Sangskolen), un collège pour enfants de dix à seize ans, est l'institution mère de plusieurs chœurs renommés, dont le Chœur de garçons de Copenhague, qui, en 2003, a reçu le prix culturel de l'Académie française ${ }^{7}$.

Bien sûr, l'accueil des lycéens après les vacances a lieu dans la salle de concert qui se remplit de plus de 700 élèves et d'une soixantaine de professeurs. Habillé en T-shirt orange, le proviseur énumère les multiples améliorations apportées à l'établissement au cours de l'été, mais sans prononcer un discours de politique générale. Parsemée de renseignements pratiques, la cérémonie d'accueil est structurée autour de deux chansons qui mobilisent l'attention et les voix des lycéens.

Les paroles d'une chanson danoise sont de Hans Christian Andersen (1805-1875), mondialement connu pour ses contes, mais également l'auteur de "Au Danemark, je suis né, c'est le pays où je suis chez moi » (I Danmark er jeg født, dér har jeg hjemme, 1850), dont tout écolier devrait connaître la mélodie lyrique. C'est en tout cas l'attente de ce lycée à l'égard de ses élèves. À l'entrée, tout le monde reçoit une partition avec l'arrangement pour quatre voix de cette chanson nationale-romantique. Ensuite, chacun est prié de se placer dans la zone qui correspond à sa voix : soprano, contralto, ténor ou basse.

Pour les élèves qui y sont habitués, ce rite collectif à l'occasion de la rentrée ne suscite plus de trouble ni de confusion ( Où dois-je aller ? Quelle voix ai-je?»). La performance se déroule sous la direction bienveillante d'un chef de chœur, à qui la tâche de conduire une telle foule ne pose aucun

7. Palsmar, p. 13. Le chœur des garçons et celui des jeunes filles font partie intégrante du collège Sainte-Anne. Le lycée, quant à lui, recrute la majorité de ses élèves parmi des candidats extérieurs, qui n'ont pas la même maîtrise chorale que celle acquise pendant les années de collège à Sainte-Anne. 


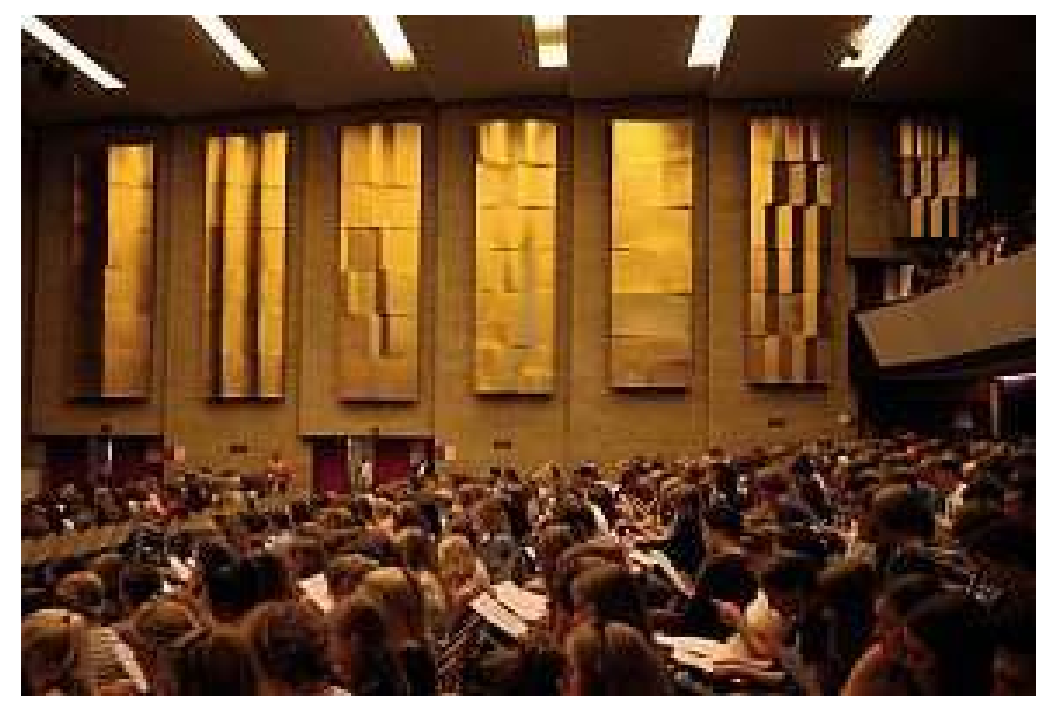

Photo 2.

Salle de concert du lycée Sainte-Anne. Rentrée 2013 (Photo : Henrik Reeh)

problème. Les différentes voix font l'objet d'un essai unique, et l'on passe à la véritable expérience musicale ; tout le monde se lève et chante debout. Personne ne reste insensible à l'intensité musicale et quasi-corporelle qu'inspire «Au Danemark je suis né (...)», chanté à quatre voix par une salle de concert entière. Cette expérience doit être forte, voire initiatrice : peu ou prou, elle fait désormais partie d'une tradition chorale qui se maintient au sein d'une culture de masse, où le déchiffrement prima vista d'une partition n'est pas à l'ordre du jour.

Est-ce afin de rassurer ceux qui se sentent moins à l'aise qu'en fin de programme, en position assise, on entonne à l'unisson With a Little Help from My Friends ( A Avec un peu d'aide de mes amis»), une chanson de Lennon et McCartney? L'accompagnement par un pianiste et par des instrumentistes à vent (majoritairement féminins), membres du big band de l'école, témoigne alors du rôle que joue la musique rythmique - jazz, rock, etc. - dans ce lycée particulier. Le principe d'entraide est certes mentionné par le proviseur, mais celui-ci laisse le public découvrir combien les paroles des Beatles se nourrissent d'un vocabulaire choral : "What would you do if I sang out of tune... (Que ferais-tu si je chantais faux) ? ", chante-t-on avant de quitter la salle au bout d'une demi-heure.

La salle de concert - dite la Salle Sainte-Anne - est un lieu public dans la vie musicale de la capitale danoise : on peut la louer et des orchestres et des chœurs s'y produisent régulièrement. En même temps, la salle fait partie intégrante de la vie pédagogique du lycée lui-même. Tous les jeudis, les élèves et les 
professeurs s'y retrouvent. Et le vendredi midi, les élèves y jouent en solistes devant leurs camarades. Enfin, c'est au balcon de la salle de concert qu'a lieu la répétition hebdomadaire des différents chœurs de l'établissement. Aussi le parterre et le balcon sont-ils convertibles en salles de conférences, équipées d'écrans de projection, etc., qui augmentent l'utilité de cet espace.

Pourtant, la salle de concert n'offre pas un lieu de passage obligé lorsque l'on se déplace à l'intérieur de l'établissement; elle en constitue un élément particulier et unique. L'état différencié de l'espace du lycée Sainte-Anne témoigne du paradigme fonctionnaliste qui assignait à chaque unité spatiale une fonction bien précise - souvent au détriment d'espaces polyvalents et unificateurs qui pourraient renforcer l'aspect urbain et communautaire du bâtiment. On touche là un point faible de l'architecture de ce lycée : une certaine urbanité semble y manquer, à l'intérieur comme à l'extérieur. Et en effet, le proviseur rêve de transférer cet établissement dans un nouveau quartier dense et multifonctionnel, au milieu de l'ancienne brasserie Carlsberg, dont le caractère monumental et labyrinthique rappelle plus la ville que la banlieue.

\section{ATRIUM ET TRANSPARENCE THÉÂTRALE AU LYCÉE ØrESTAD}

Depuis l'achèvement du lycée Sainte-Anne en 1972, il a fallu attendre trente-cinq ans pour assister à l'inauguration d'un nouveau lycée dans la municipalité de Copenhague, plus précisément à Ørestad, ville nouvelle phare qui se situe entre la ville historique et l'aéroport international. En 2007, le lycée Ørestad a ouvert ses portes à l'endroit stratégique où se croisent le métro, les voies ferrées internationales, l'autoroute vers la Suède et un boulevard urbain. L'architecture spectaculaire de ce lycée est due aux architectes 3XN qui, en 2013, ont achevé l'importante Cité des Nations unies à Copenhague. Entouré d'un centre commercial, d'immeubles de bureaux et d'appartements, ainsi que d'un parc naturel protégé, le lycée Ørestad s'inscrit dans un plan d'urbanisme dont la linéarité et les discours de croissance rappellent les années 1960 et 1970, l'âge d'or du fonctionnalisme. Or une importance nouvelle est accordée à l'architecture signalétique et au contexte urbain, ainsi qu'au discours sur le développement durable, d'où les sommes considérables investies dans des institutions civiques telles que le lycée Ørestad.

Malgré ses volets colorés, fort visibles depuis l'extérieur, c'est par son intérieur que le lycée Ørestad fait date dans l'histoire de l'architecture scolaire et urbaine. Si ce n'était pour la présence des élèves, on se croirait dans un théâtre, un grand magasin ou dans l'atrium d'une grande entreprise ${ }^{8}$, soucieuse de la fonction communicationnelle de son architecture. En effet, l'espace du lycée se présente comme un immense lobby, où un escalier sculptural s'élève en

8. Missac, $185-210$. 
spirale à travers les cinq étages du volume intérieur. À chacun des étages supérieurs, on trouve, certes, des salles de classe, mais celles-ci s'intègrent à l'espace communautaire par leurs parois en verre transparent. Tout visiteur peut voir comment les corps des élèves s'orientent par rapport à l'écran de projection qui a remplacé le tableau noir d'antan. En ce jour de rentrée, les présentations PowerPoint révèlent les efforts des professeurs pour expliquer aux élèves combien leur présence assidue est obligatoire s'ils veulent garder leurs bourses d'État et, non moins important, leur droit de s'inscrire aux examens du baccalauréat. Ce lycée a connu de graves problèmes d'absentéisme, raison pour laquelle une discipline de ponctualité est mise en place dès le début de l'année scolaire.

Cet établissement se veut le lycée de l'avenir pédagogique, technologique et architectural, en bref, le lycée de la société d'information et de créativité qui semble remplacer celui de la modernité industrielle et sociale ${ }^{9}$, dont relèvent les deux autres lycées étudiés. Sur le site Internet du lycée Ørestad, une élève de première assure n'avoir jamais eu un stylo à la main; à la place, chaque élève reçoit un iPad en prêt gratuit afin de compléter son ordinateur personnel, de préférence un iBook d'Apple. Car les produits Apple sont compatibles avec l'équipement informatique omniprésent, disponible pour les élèves pendant les heures de cours, bien sûr, mais aussi l'après-midi, quand ils font leurs devoirs dans les zones de travail ou de récréation aménagées à tous les étages de l'atrium. Il suffit de s'y installer - seul ou en groupe. Parmi les séries et les enseignements de spécialité proposés aux lycéens, le design et les médias occupent une place privilégiée, ce qui va de pair avec la demande soutenue pour les 400 places disponibles par an, presque le double des promotions des lycées Øregård et Sainte-Anne. De par son architecture, son équipement informatique et ses orientations pédagogiques, le lycée Ørestad tente de franchir le pas vers le lycée de masse. C'est un défi tout à fait réel à une époque où plus de $40 \%$ des jeunes fréquentent le lycée général - dix fois plus qu'à l'achèvement du lycée Øregård pendant l'entre-deux-guerres, et le double du nombre déjà élevé de lycéens lors de l'ouverture du lycée Sainte-Anne en 1972. Autour de l'année 2000, une nouvelle période de forte croissance a commencé ${ }^{10}$.

La tentative pour adapter les stratégies pédagogiques à un recrutement qui va bien au-delà des milieux sociaux privilégiés et des critères de la tradition musicale et académique se traduit dans les activités proposées à la rentrée. Ici, rien ne serait plus étranger au programme que le chant à plusieurs voix. Si un professeur projette effectivement, à l'écran, les paroles lyriques d'une chanson danoise très connue, "Dans le calme silencieux et profond de la forêt » (I skovens dybe stille ro, 1864), son intention n'est pas de mobiliser les voix des élèves. Ce texte lyrique lui sert, au contraire, à illustrer ce que le nouveau lycée,

9. Bertelsen, 134-135.

10. Les chiffres de la croissance actuelle sont disponibles sur : http://www.uvm.dk/Service/Statistik/Statistik-omgymnasiale-uddannelser/Elevtal-for-de-gymnasiale-uddannelser 
promouvant la culture contemporaine, tente de dépasser. La chanson est citée comme un exemple étranger à la jeunesse lycéenne, alors que l'enregistrement en 1973 par le contrebassiste de jazz Niels Henning Ørsted Pedersen et le pianiste Kenny Drew avait fait de cette mélodie populaire un tube, voire un nouveau classique.

La rentrée des élèves se fait selon un scénario qui se révèle être la négation de ceux des lycées Øregård et Sainte-Anne. À Ørestad, les lycéens de première et de terminale ne sont pas exposés à la parole du proviseur ; la modération de la cérémonie d'accueil est assurée par deux élèves qui invitent leurs camarades à une animation qui implique, notamment, le spectacle d'un comédien professionnel, connu à la télévision nationale.

L'agencement spatial de ce spectacle collectif se distingue nettement de ce que nous avons vu dans les deux autres établissements scolaires. Ici, ni l'aula centrale d'Øregård ni la salle de concert pour mille personnes du lycée Sainte-Anne n'encadrent la position corporelle et l'attention réceptive des lycéens. Puisque l'atrium d'Ørestad est dépourvu de scène proprement dite, la performance a lieu sur le sol même de la salle de gymnastique du sous-sol; les cloisons amovibles permettent d'ouvrir le gymnase vers l'étage inférieur de l'atrium. Ainsi les spectateurs sont-ils assis par terre dans la salle de sport ou installés soit sur les grandes marches de l'atrium, soit sur les chaises qui, au quotidien, servent aux élèves pour travailler dans les zones communes du lycée. Il y a même des spectateurs debout le long des balustrades des étages supérieurs.

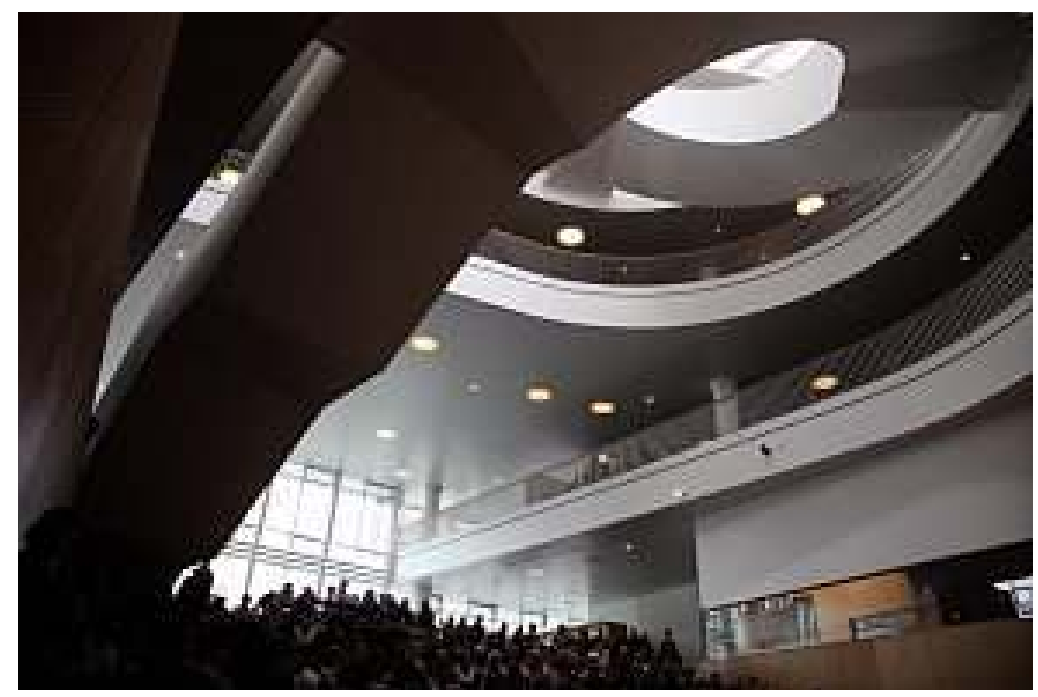

Photo 3.

Atrium du lycée Ørestad. Rentrée 2013 (Photo : Henrik Reeh) 
Pendant l'accueil de rentrée, l'attention des lycéens est orientée vers le bas des marches, où se trouve le comédien. Mais les regards d'en haut vers les étages du bas dominent aussi la visualité quotidienne de cet atrium dramatique et à délimitation diffuse. Même si, à chaque étage, on peut voir des gens derrière les parois transparentes, les regards des élèves et des professeurs s'orientent surtout verticalement, en suivant la courbe de l'escalier sculptural qui mène du rez-de-chaussée au toit-terrasse.

L'architecture du récent lycée Ørestad ne se limite pas à reprendre le rôle symbolique et spatial joué par l'aula intérieure du lycée Øregård. À Ørestad, l'atrium central est visible de la rue et du métro aérien. Vu la transparence des salles de classes mais aussi des bureaux des professeurs et même de la direction, il ne reste guère que quelques salles de projection qui soient fermées aux regards extérieurs. Ce lycée exhibe un espace scolaire qui nourrit les fantasmes de la transparence, de la flexibilité et de la théâtralité.

Les espaces scolaires du lycée dépassent de loin les salles de classe où se fait la transmission des connaissances les plus immédiates. C'est la raison pour laquelle il ne faut pas sous-estimer les espaces communs de chaque établissement : couloirs, escaliers, cantines, cours de récréation, etc. Tous les lycées, sans exception, possèdent une salle des fêtes où ont lieu les cérémonies symboliques, dont la très festive cérémonie de fin d'études, à la fin des trois années menant au baccalauréat, mais aussi les représentations de théâtre et de musique, où les élèves montent sur scène.

Comparé aux spectacles officiels, qui s'adressent en grande partie aux parents, l'accueil des élèves après les vacances d'été a un caractère interne. L'accueil est néanmoins un rite de passage, et c'est un temps fort dans l'expérience de tout élève qui, à cette occasion, semble grandir d'une année entière; cela n'arrive que deux fois au cours d'une scolarité lycéenne qui, au Danemark, va de seize à dix-neuf ans. Tout en s'appuyant sur le cadre spatial et sur la particularité des élèves inscrits, la direction se sert aussi - plus ou moins consciemment - de ce moment intense pour mettre en scène une vision adéquate de l'établissement.

Représentants de trois phases de l'État-providence, les lycées Øregård (1924), Sainte-Anne (1972) et Ørestad (2007), tous trois situés à Copenhague, ont des salles des fêtes qui résument le destin de l'architecture scolaire (en particulier lycéenne) au cours du XXe et au début du XXI ${ }^{\mathrm{e}}$ siècles. À cet égard, le rôle primordial joué par l'aula du lycée Øregård n'est pas une exception architecturale. À la fois variable et transposable, le type architectural de l'aula a survécu à maintes transformations pédagogiques et esthétiques depuis un siècle. Parfois l'espace communautaire inauguré par l'aula s'empare du quasi-ensemble de 
l'architecture scolaire. Dans le récent lycée Ørestad, la transparence du hall s'étend jusque dans les salles de classe les plus spécialisées. Cependant, la salle de concert du lycée Sainte-Anne témoigne de la différenciation spatiale propre à l'époque fonctionnaliste - différenciation qui semble céder le pas à une spatialité plus diffuse et flexible louée par le post-fordisme néo-libéral. Or, comme l'on a vu, même une salle de concert se prête à une multitude d'usages, musicaux ou non.

L'examen des trois lycées danois montre combien l'accueil des lycéens à la rentrée inspire une utilisation variée des espaces scolaires partagés. Certes, il n'est guère surprenant que l'aula néoclassique d'Øregård soit le lieu privilégié du discours du proviseur, ni que la salle de concert propre à l'architecture fonctionnaliste du lycée Sainte-Anne permette à la communauté scolaire de fusionner dans le chant à quatre voix. Néanmoins, la forme architecturale n'impose pas une unique stratégie pédagogique ; il reste une marge de manœuvre qu'il faudrait prendre au sérieux. Ainsi l'atrium du lycée Ørestad pourrait-il servir à des formes d'accueil qui continueraient et renouvelleraient la tradition démocratique et l'expression culturelle promues par les autres exemples d'architecture scolaire. Beaucoup dépend de la stratégie culturelle des directions d'établissement mais aussi des contributions apportées par les enseignants et, ne l'oublions pas, par les élèves.

\section{BIBLIOGRAPHIE}

ASKGAARD Helle, HAUGAN Kamma (ed.) (2003) : Tidsbilleder: Plockross' Skole og Øregård Gymnasium. Hellerup : Øregård Gymnasium.

ASKGAARD Helle, HAUGAN Kamma (2011) : « Øregård Gymnasium ». In N. de Coninck-Smith, Harriet Tranum-Jensen (ed.) : 2900 Hellerup - set i bakspejlet, 64-83. Charlottenlund : Strandbergs Forlag.

BENJAMIN Walter (2000 [1935/1936]) : «L'œuvre d'art à l'époque de sa reproductibilité technique », trad. par Rainer Rochlitz. In W. Benjamin : Euvres, t. III. Paris : Gallimard, p. 67-113. (Voir aussi W. Benjamin : Gesammelte Schriften, t. VII, 350-384, 661-609. Francfort sur le Main : Suhrkamp Verlag, 1989.)

BERTELSEN Eva (2013): 'Curriculum' til fremtiden? Karakteristikker af 'den nye gymnasieskole’ gennem perspektiver på Institution, nybyggeri og elevsubjektiviteter. Ørestad Gymnasium som case. Thèse de doctorat, Université de Copenhague, Faculté des Sciences Humaines.

CONINCK-SMITH Ning (2011): Barndom og arkitektur: Rum til danske børn igennem 300 år. Aarhus : Klim.

HANSEN Preben : "Sankt Annæ Gymnasiums koncertsal, Valby". In Arkitektur DK, vol. 19, n 4, 1975, p. 164-168, avec des résumés en anglais et en allemand.

JØRGENSEN Lisbet Balslev, LUND Hakon \& NØRREGÅRD-NIELSEN Hans Edvard (1985) : Danmarks arkitektur - Magtens bolig. Copenhague : Gyldendal.

MISSAC Pierre (1987) : Passage de Walter Benjamin. Paris : Seuil. 
PALSMAR Henrik (ed.) : For Skolen og for Livet. Om korsang, dannelse og loereprocesser. Festskrift 75 år. Sangskolen på Sankt Annoe Gymnasium. Copenhague : Danmarks Pædagogiske Universitets Forlag.

REEH Henrik (2006) : "Blik i brug - Walter Benjamin og erfaringens arkitektur" in C. Thau (ed.) : Filosofi \& Arkitektur i det 20. århundrede. Copenhague : Kunstakademiets Arkitektskole [Académie Royale des Beaux-Arts, École d'Architecture], p. 83-124. 